Journal of Computer Science 6 (2): 168-172, 2010

ISSN 1549-3636

(C) 2010 Science Publications

\title{
An Algorithm for Localization in Vehicular Ad-Hoc Networks
}

\author{
Hajar Barani and Mahmoud Fathy \\ Department of Computer Engineering, Iran University of Science and Technology, Tehran, Iran
}

\begin{abstract}
Positioning a node in Vehicular Ad-Hoc networks is one of the most interested research areas in recent years. In many Ad-Hoc networks such as Vehicular Ad-Hoc networks in which the nodes are considered as vehicles, move very fast in streets and highways. So, to have a safe and fast transport system, any vehicle should know where a traffic problem such as a broken vehicle occurs. GPS is one of the equipments which have been widely used for positioning service. Problem statement: Vehicle can use a GPS receiver to determine its position. But, all vehicles have not been equipped with GPS or they cannot receive GPS signals in some places such as inside of a tunnel. In these situations, the vehicle should use a GPS free method to find its location. Approach: In this study, a new method based on transmission range had been suggested. Results: This algorithm had been compared with a similar algorithm ODAM in same situations. The best performance for Optimized Disseminating Alarm Message (ODAM) is when $40 \%$ of nodes are equipped with GPS. Conclusion: We executed our algorithm on this situation and compared it with ODAM results. At the end of this study, we can see our algorithm in compare to ODAM has better results.
\end{abstract}

Key words: Vanet, localization, GPS equipped nodes

\section{INTRODUCTION}

In Vehicular Ad-Hoc Network (VANET), nodes which are vehicles can move with very high speed and generally must communicate quickly and reliably. When an accident occurs in road or highway, an alarm message must be disseminated, instead of Ad-Hoc routed, to inform all other vehicles. Positioning a broken vehicle and locating the vehicles in its vicinity is very important for the safety of the road users. The most popular method for localization is the GPS. The Global Positioning System (GPS) was first proposed in the 1960's by the US Navy and the first working prototype was developed in 1978 (HofmannWellenhof et al., 2001). GPS was deployed based on the NAVSTAR satellite constellation in 1993 (Kaplan, 1996). It uses single-hop positioning system to coverage the position and also, uses Time Difference Of Arrival (TDOA) technology to localize itself via several anchor nodes directly (Caffery and Stuber, 1998). The most important problem with the GPS is that it can not receive signals inside buildings, underground or in tunnels. Also, vehicles are not necessarily equipped with GPS and even they can not obtain availability of line of sight access to satellites, particularly when they enter tunnels (Benslimane, 2005).
The Wireless Fidelity (WiFi) provides the navigation, tracking function in the big campuses area like universities and airports. One of the projects which used these methods is Vehicle Tracking (VETRAC) (Thangavelu et al., 2007). A navigation system is a radio frequency based wireless mobile system which designed to guide vehicles in its location using digital maps display (Blum et al., 2004; Ye et al., 1998; Bostrom et al., 2002).

Also, in such a situation, vehicles can use a localization technique to overcome this problem. Lots of localization algorithms have been proposed in AdHoc networks.

The basis of different localization algorithms in the literature are consist of two main steps:

- A ranging procedure which involves estimating the distance from a sensor to another sensor based on the signal strength of the signals transmitted/received between the two

- A procedure that recovers the locations of the sensors based on their pair wise distance estimates either by triangulation or by least-squares methods (Priyantha et al., 2000; Savvides et al., 2001; Girod and Estrin, 2001).

Corresponding Author: Hajar Barani, Department of Computer Engineering, Iran University of Science and Technology, Tehran, Iran Tel/Fax: 0098-341-247-2461 
In most of these algorithms the distance between GPS-equipped nodes with GPS-unequipped nodes should obtained. Then with the help of distance and the location of GPS-equipped nodes the location will approximated. These algorithms can find the distance with two techniques. First, they can work on Radiolocation method and second, they can work on topology-based methods. As we use a radio location method, we will have a discussion on the Radiolocation methods.

\section{MATERIALS AND METHODS}

Radiolocation methods: There are several radio location methods which apply signal measurements to determine the direction of the paths between a node and the base station (Benslimane, 2005). But the main three methods are used today: Received Signal Strength Indicator (RSSI), Time of Arrival (TOA), Time Difference of Arrival (TDOA) and Angle of Arrival (AOA).

RSSI: This method measures the power of signal at the receiver. The effective propagation loss can be computed based on the known transmit power. In this method, the node must lie on a circle which the BS is on its center. At least three of these BSs needed for computing the nodes position. Then the distance will be calculated according to this equation (Wesel, 1997):

$$
P_{r}=P_{t}\left(c_{1} / d\right)^{n} \cdot c_{2}
$$

\section{Where:}

$\mathrm{P}_{\mathrm{t}} \quad=$ The power level on which the message is sent

$\mathrm{P}_{\mathrm{r}} \quad=$ The power level on which the message is received

$\mathrm{n}, \mathrm{c}_{1}, \mathrm{c}_{2}=$ Some environmental variables (HofmannWellenhof et al., 2001)

TOA, TDOA: This technique is based on measuring the TOAs which were received at several BSs or the Time Difference Of Arrival (TDOA) of a signal received at multiple pairs of BSs. In the TOA approach, the distance between an MS and a BS is measured by finding the one-way propagation time between an MS and a BS. Geometrically, this provides a circle, centered at the BS, on which the MS must lie. Refer to previous sections ambiguities, the MS's position is given by the intersection of the circles (Benslimane, 2005).

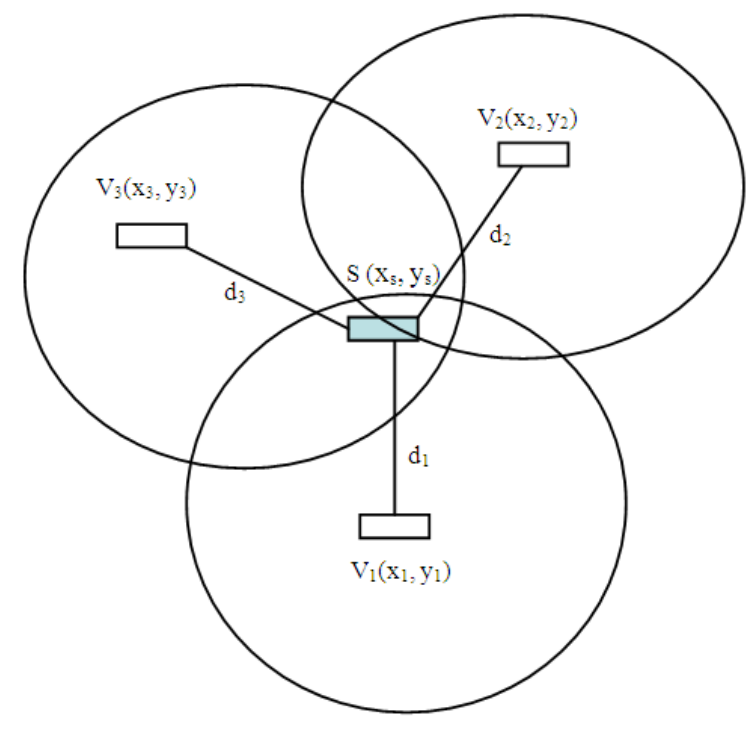

Fig. 1: Locating a node with three neighbors

AOA: AOA techniques estimate the MS location by first measuring the AOAs of a signal from an MS at several BSs through the use of antenna arrays. Scattering near and around the MS and BS will alter the measured AOA. In the absence of an LOS signal component, the antenna array will lock on to a reflected signal that may not be coming from the direction of the MS. Even if an LOS component is present, multipath will still interfere with the angle measurement. The accuracy of the AOA method diminishes with increasing distance between the MS and BS due to fundamental limitations of the devices used to measure the arrival angles as well as changing scattering characteristics (Benslimane, 2005).

After measuring the distance another method which named "Triangulation" will be used to estimate the location. This method used when estimating the direction of the node is important, as in AOA systems. After that the position of node can be calculated by using the trigonometry laws of sines and cosines (Savvides et al., 2001).

GPS-free algorithm: Assume node $\mathrm{S}$ wants to find its location. This node sends a broadcast message to its neighbors $\left(\mathrm{v}_{1}, \mathrm{v}_{2} \ldots\right)$ with a constant transmission range. When neighbors receive the broadcast message, they would check if they know their location via GPS or localization algorithm. If know it, they answer $\mathrm{S}$ with a message contain their location. Node S, counts the answers received. If it has received at least three answers from nodes $\mathrm{v}_{1}\left(\mathrm{x}_{1}, \mathrm{y}_{1}\right), \mathrm{v}_{2}\left(\mathrm{x}_{2}, \mathrm{y}_{2}\right), \mathrm{v}_{3}\left(\mathrm{x}_{3}, \mathrm{y}_{3}\right)$, then the location can be estimated with triangulation formula (Fig. 1): 
$\left(\mathrm{x}_{\mathrm{s}}-\mathrm{x}_{1}\right)+\left(\mathrm{y}_{\mathrm{s}}-\mathrm{y}_{1}\right)=\mathrm{d}_{1}$

$\left(\mathrm{x}_{\mathrm{s}}-\mathrm{x}_{2}\right)+\left(\mathrm{y}_{\mathrm{s}}-\mathrm{y}_{2}\right)=\mathrm{d}_{2}$

$\left(\mathrm{x}_{\mathrm{s}}-\mathrm{x}_{3}\right)+\left(\mathrm{y}_{\mathrm{s}}-\mathrm{y}_{3}\right)=\mathrm{d}_{3}$

But, there are not always three neighbors around node $\mathrm{S}$ which equipped with GPS. Our algorithm uses a new method to find more neighbors, thus it can estimate its location in more situations.

In our algorithm, node $\mathrm{S}$ broadcasts a request message for estimating the location.

When node $\mathrm{v}(\mathrm{x}, \mathrm{y})$ receives the request from node $\mathrm{S}$, it broadcast this request again. With this rebroadcast, more GPS-equipped nodes will be discovered. Then, node $\mathrm{S}$ collects the data received from other nodes. If three nodes are in one-hop distance from node $S$, it will ignore the other two-hop neighbor's locations.

If there are two nodes in one-hop neighboring, node $\mathrm{S}$ uses the two-hop neighbor for finding its location. Node $\mathrm{S}$ sends a signal to this node and estimates the distance between them. Then with the help of this distance the location will be calculated (Fig. 2).

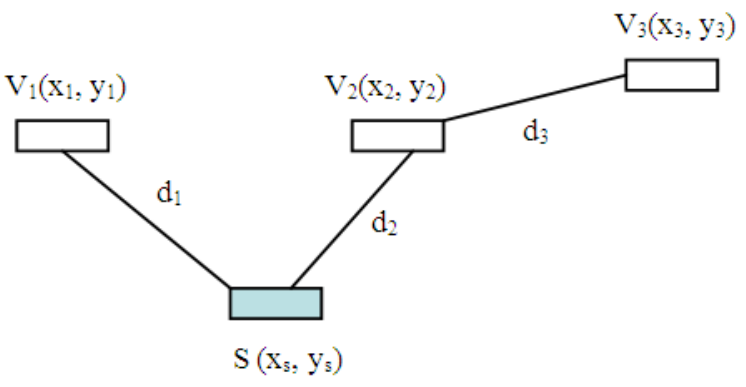

Fig. 2: Locating a node with two one-hop nodes

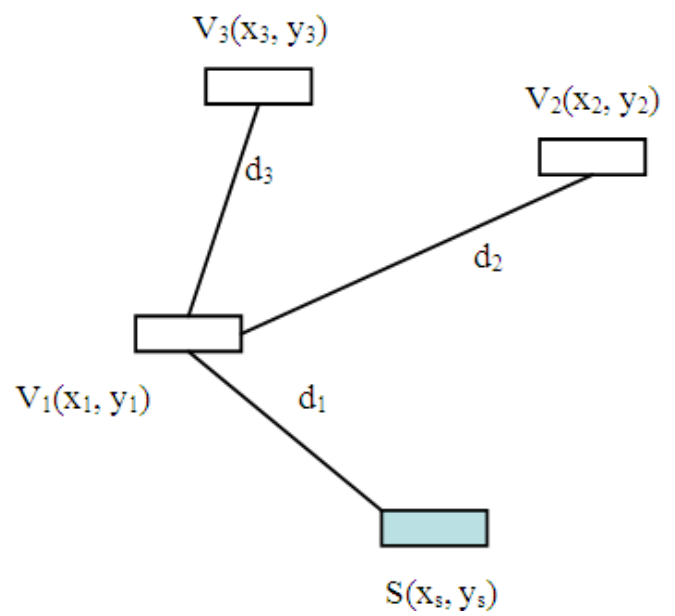

Fig. 3: Locating a node with one one-hop node
When there is only one neighbor (node V) in onehop neighboring from $\mathrm{S}$, it should find two nodes in its neighborhood. If it finds them, node $S$ uses these three nodes to estimate its location. But in this situation the location will be estimated with a little delay (Fig. 3).

In the other situations the location can not be calculated, because there will not be three neighbors in its one or two hop neighborhood. So, node S should try for the algorithm some moments later.

\section{RESULTS AND DISCUSSION}

This algorithm has been simulated in with Glomosim simulator. In this simulation we use 150 nodes in $20 \mathrm{~km}$ two way road. Vehicles are moving in different speed in their line.

As we know, the location of the node can be estimated when it has at least three GPS-equipped neighbors. Thus, for estimating the performance of the node we just count the neighbors of node $S$. If they are more than three then the location will be obtain. In here we use different densities to calculate the algorithm in different situations. Also, the percent of GPS-equipped nodes in whole environment is important, because the location of the node is estimated based on the GPSequipped nodes.

With $20 \%$ of GPS-equipped nodes and different transmission Range (R) we calculate the GPS-equipped nodes. In this situation the GPS-equipped nodes will not be equal to three even with high transmission rang and highest density till the transmission range is 400 which is the highest range in our localization algorithm and the density of $80 \%$ (Fig. 4).

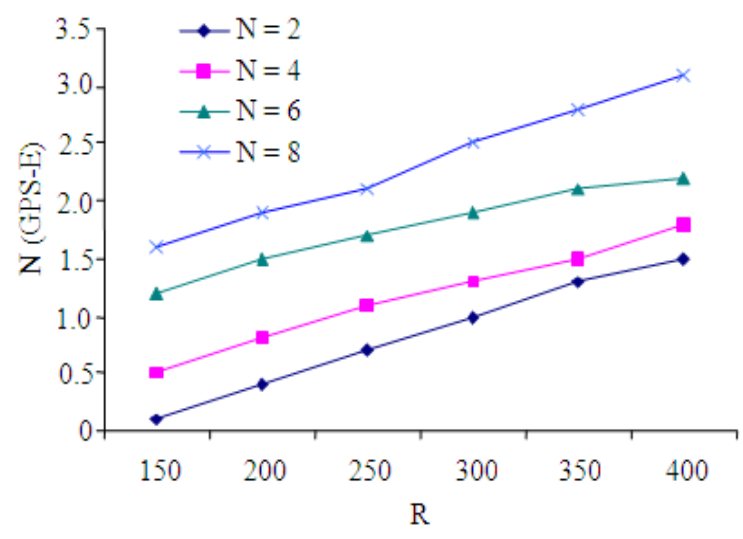

Fig. 4: GPS-E = 20\% 


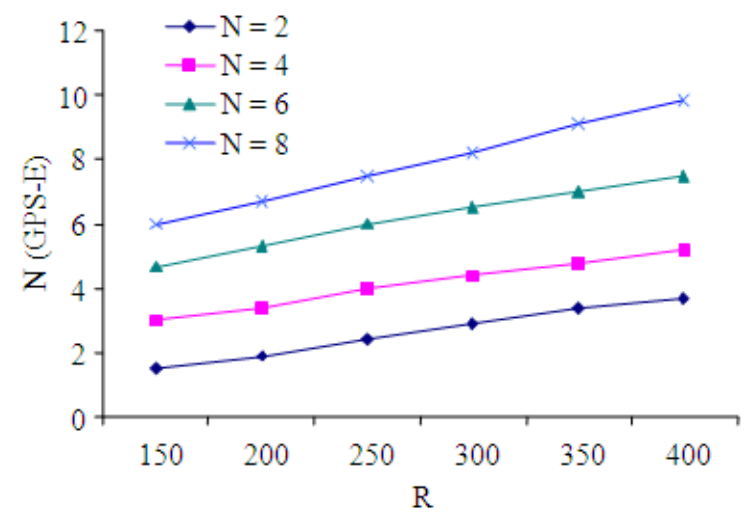

Fig. 5: GPS-E $=40 \%$

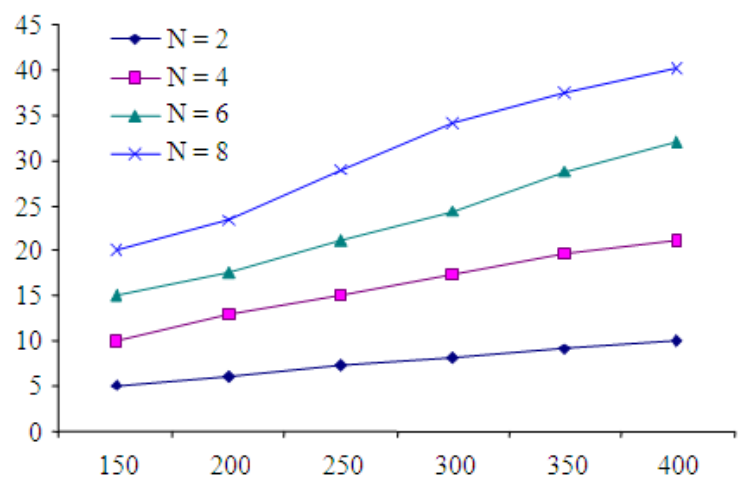

Fig. 6: GPS-E $=80 \%$

In the next situation, we increase the GPSequipped nodes to $40 \%$ of the whole nodes. In here we can see that the number of GPS-equipped neighbor is more than three in most cases. So, just for the lowest transmission range and GPS-equipped nodes in whole environment and also, low density $(\tau=0.2)$ the location will not discovered (Fig. 5). In result, this is a good situation for nodes to use this algorithm and find their location.

In last situation, the GPS-equipped nodes increased to $80 \%$ of whole nodes. In this situation, with different density and different transmission range at least the locations of three nodes are obtained. So, in all situations the location of the nodes can be calculated (Fig. 6).

In the other situations based on the percentage of GPS-equipped nodes, some neighbors will be discovered. But they are not suitable anymore. The performance of this algorithm is optimal even with less than $40 \%$ initially GPS-E vehicles.

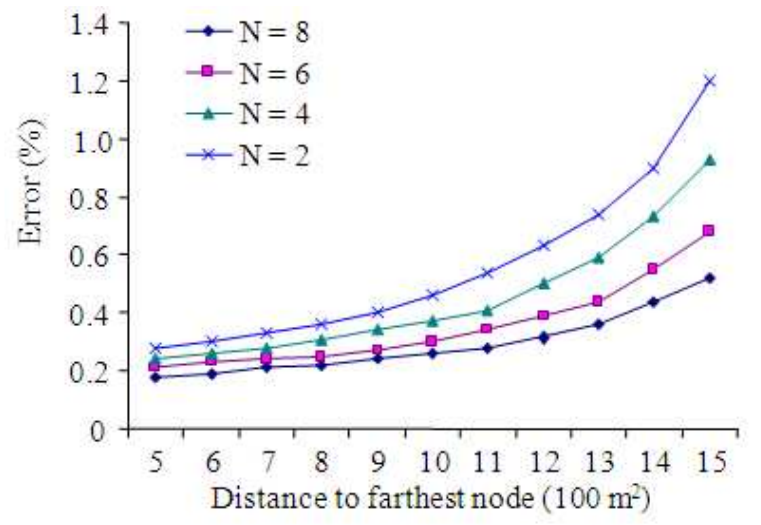

Fig. 7: Error in algorithm

\section{CONCLUSION}

After calculating all the GPS-equipped neighbors of the node, we can see that the best situation for the nodes is when the number of GPS-equipped nodes are around $40 \%$ of all nodes. But in compare of ODAM method, this method has better answers. In ODAM with GPS-E $=0.2$ nodes will never find their location. But in this method with high density and transmission range, we will have an answer. And in compare of ODAM localization method, in other situations with different densities our algorithm works better.

Also, in this method, because of having some calculations during the algorithm the location will be obtained after a short time. So, the location will estimate with a little difference with the exact position. This error is depending on the distance between two nodes. Also, the error of the algorithm is based on the density of nodes (Fig. 7).

As a future research, decreasing this error is a good project to work on. Also, finding a way to calculate distances in a short time is an interesting area for research.

\section{REFERENCES}

Benslimane, A., 2005. Localization in vehicular ad-hoc networks. Proc. Syst. Commun., 1: 19-25. DOI: 10.1109/ICW.2005.54

Blum, J., A. Eskandarian and L. Hoffman, 2004. Challenges of intervehicle ad-hoc networks. IEEE Trans. Intel. Transport. Syst., 5: 347-351. DOI: 10.1109/TITS.2004.838218

Bostrom, T., T. Goldbeck-Lowe and R. Keller, 2002. Ericsson mobile operator WLAN solution. Ericsson Rev., 78: 36-43. 
Caffery, J.J. and G.L. Stuber, 1998. Overview of radiolocation in CDMA cellular systems. IEEE Commun. Mag., 36: 38-45. DOI: 10.1109/35.667411

Girod, L. and D. Estrin, 2001. Robust range estimation using acoustic and multimodal sensing. Proceeding of the IEEE/RSI International Conference on Intelligent Robots and Systems, Oct. 29-Nov. 3, IEEE Xplore Press, Maui, Hawaii, USA., pp: 1312-1320. DOI: 10.1109/IROS.2001.977164

Hofmann-Wellenhof, B., H. Lichtenegger, and J. Collins, 2001. GPS: Theory and Practice. 5th Revised Edn., Springer, USA., ISBN: 978-3211835340, pp: 370.

Kaplan, E.D., 1996. Understanding GPS Principles and Applications. Artech House Publishers, ISBN: 10: 0890067937, pp: 576.

Priyantha, N.B., A. Chakraborty, and H. Padmanabhan, 2000. The cricket location support system. Proceeding of the 6th Annual International Conference on Mobile Computing and Networking, Aug. 6-11, ACM Press, Boston, Massachusetts, United States, pp: 32-43. DOI: 10.1145/345910.345917

Savvides, A.. C.C. Han and M. Srivastava, 2001. Dynamic fine-gained localization in ad-hoc networks of sensors. Proceeding of the 5th International Conference on Mobile Computing and Networking, (MCN'01), ACM Press, Rome, Italy, pp: 166-179. DOI: 10.1145/381677.381693
Thangavelu, A., K. Bhuvaneswari and K. Kumar, 2007. Location identification and vehicle tracking using VANET (VETRAC). IEEE Int. Conf. Sign. Proc. Commun. Network., 1: 112-116.

Wesel, E.K., 1997. Wireless Multimedia Communications: Networking Video, Voice and Data. 1st Edn., Addison-Wesley, Massachusetts, USA., ISBN: 10: 0201633949, pp: 298.

Ye, T., H. Jacobsen and R. Katz, 1998. Mobile awareness in a wide area wireless network of infostations. Proceeding of the 4th Annual ACM/IEEE International Conference on Mobile Computing and Networking, Oct. 25-30, ACM Press, Dallas, Texas, USA., pp: 109-120. DOI: $10.1145 / 288235.288264$ 